\title{
A simple model for viscous regularization of elasto-plastic constitutive laws with softening
}

\author{
V. Dias da Silva*,† \\ Department of Civil Engineering, University of Coimbra, Polo II da Universidade-Pinhal de Marrocos, \\ P-3030-290 Coimbra, Portugal
}

\begin{abstract}
SUMMARY
An overlay-type rheological model is presented, which is used to introduce viscosity in inviscid elastoplastic material laws with softening, in order to reduce the mesh-dependency of Finite Element solutions. This model is intended to be an alternative to the well-known visco-plastic formulations of Perzyna and Duvaut-Lions. A time integration algorithm for the visco-elastic model component is presented, being demonstrated in the paper, that it is unconditionally stable and oscillation-free. The algorithm is tested in a problem with slip driven softening (von Mises material) and in a problem with decohesion driven softening (Cam-Clay model). Figures showing the capability of the algorithm to regularize the solution are presented. Copyright (c) 2004 John Wiley \& Sons, Ltd.
\end{abstract}

KEY WORDS: softening; regularization; visco-elasticity; elasto-plasticity; Cam-Clay; von Mises

\section{INTRODUCTION AND OVERVIEW}

Constitutive laws with softening — as, for example, the Cam-Clay elasto-plastic model-lead to mesh-dependent results, when used with finite element discretizations. From a physical point of view, this mesh dependency is a consequence of the fact that the finite element description of the continuum is not perfect, but allows for stress discontinuities at the element boundaries. As a consequence of this, the deformation concentrates in a band of finite elements, whose width and direction depend on the used mesh.

The more usual ways to regularize the solution, i.e. to avoid or, at least, to reduce the mesh dependency, are [1]:

1. the use of a rotation sensitive material law, as the Cosserat continua;

2. the non-local concept, in which the hardening parameters have a non-local definition, leading to gradient plasticity theories;

3. the introduction of viscosity in the material law.

\footnotetext{
${ }^{*}$ Correspondence to: V. Dias da Silva, Depto de Engenharia Civil, Universidade de Coimbra, Polo II da Universidade, Pinhal de Marrocos, P-3030-290 Coimbra, Portugal.

†E-mail: vdsilva@dec.uc.pt
} 


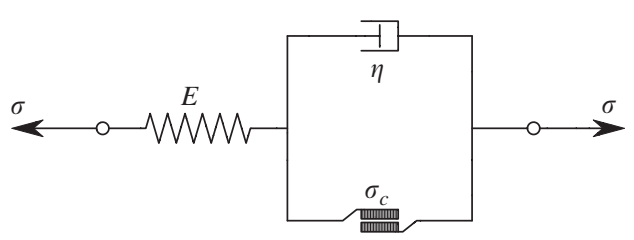

Figure 1. Rheological model on which the formulations of Perzyna and Duvaut-Lions are based.

The first possibility furnishes good results for grain boundary sliding plasticity. However, for decohesion driven softening, the results are poor. Besides, for its use, it is necessary to program a finite element procedure, which includes the additional degrees of freedom (the rotations).

The second possibility - gradient plasticity — is highly versatile and works equally well for both slip and decohesion softening [1]. Its main disadvantage is the introduction of an additional variable, which must be discretized at the global level.

The third possibility - the rate-dependent behaviour induced by the viscosity - is based on the fact that localization causes high deformation rates, which are reduced and distributed in the finite element mesh by means of the viscosity. Its main disadvantage is the necessity of adding an artificial feature - the viscosity - to the material behaviour, when it does not display rate dependence. Its main advantage is that it does not need any additional global discretizations, since it requires only supplementary operations at the local level (constitutive law), whose implementation in common non-linear finite element packages is very simple. Furthermore, it works equally well, both for decohesion and slip driven softening.

For the inclusion of viscosity in the elasto-plastic constitutive law the models of Perzyna and Duvaut-Lions [2-5] have been used. More recently Wang et al. [5] have introduced a model, in which the viscosity is implemented by means of a rate-dependent yield surface.

The model proposed in the present contribution is intended to be an alternative to the formulations of Perzyna and Duvaut-Lions, which are based on the same one-dimensional model, cf. Figure 1 [6]. The major drawback of the model of Perzyna is the fact that it may not converge to the inviscid solution, when the viscosity goes to zero, in non-smooth multi-surface plasticity [4]. The Duvaut-Lions model has the limitation that it must be used in conjunction with an integration algorithm for the inviscid elasto-plastic rate equations, in which the initial stress is used only to compute the trial stress, since it may be outside the yield surface. Usually a backward Euler interpolation is used. Furthermore, an evolution rule must be postulated for the yield surface, in case of hardening/softening plasticity [4]. The model proposed in this work was designed to avoid these limitations, i.e. to be compatible with any integration schema for the inviscid rate equations and to automatically converge to the inviscid solution, when the viscosity goes to zero.

The model presented here combines a Maxwell-type visco-elastic behaviour with a generic elasto-plastic rheological model. The procedure is then implemented on the example of an elasto-plastic von Mises constitutive law with (slip driven) softening and also with a CamClay elasto-plastic model. The latter rheological model follows a volumetric hardening scheme, which automatically includes decohesion driven softening. 
In the described examples, the elasto-plastic part of the material response is computed via a backward Euler algorithm (radial return), while for the visco-elastic part an unconditionally stable and oscillation-free mid-point indirect interpolation scheme is used.

After description of the analytical and numerical tools used, results from the finite element simulation of the process of formation of shear bands in material specimens, using both elasto-plastic constitutive laws on different FE-meshes, are presented and discussed.

\section{THE MODEL}

\subsection{Outline}

In classical displacement based finite element programs the elasto-plastic constitutive law is usually implemented in a routine, in which, for a given strain increment, the stress increment and the material stiffness are computed. When a complex material behaviour is to be modelled, it is therefore convenient to use a material model of the overlay type, as the one represented in Figure 2, in the one-dimensional case. This model is composed of an elastoplastic element $\left(E_{0}, \sigma_{c}\right)$ and a Maxwell visco-elastic element $(E, \eta)$. In its generalization to the three-dimensional case, the strain tensor is the same in both elements. Therefore, for given strains, stresses and stiffness can be computed separately for each element and added. The elasto-plastic elements used, as examples, in the present paper are, as referred, the von Mises and the Cam-Clay models.

\subsection{The visco-elastic Maxwell model}

The Maxwell model consists of a serial assembly of an elastic element (spring) of stiffness $E$ and a viscous element (dashpot), whose coefficient of viscosity is $\eta$. The constitutive law of the model reads

$$
\dot{\varepsilon}=\frac{\sigma}{\eta}+\frac{\dot{\sigma}}{E}
$$

where $\sigma$ is the stress in the Maxwell model component and the dot denotes, as usually, the time derivative. In order to have the advantages of an indirect approximation of the inelastic strain, in what concerns stability and accuracy of the time integration, the constitutive law must be used in integral form [7], which is obtained by solving the differential equation (1), yielding

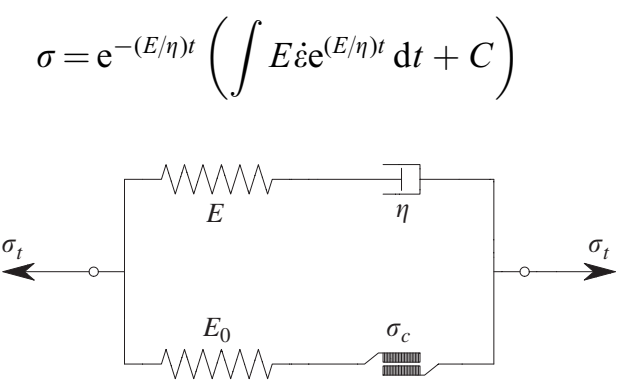

Figure 2. Proposed overlay model. 
The integration constant $C$ may be eliminated by considering an initial stress $\sigma_{0}$ at the time $t_{0}$, yielding

$$
\sigma(t)=\sigma_{0} \mathrm{e}^{-(E / \eta)\left(t-t_{0}\right)}+\int_{t_{0}}^{t} E \dot{\varepsilon} \mathrm{e}^{-(E / \eta)(t-\tau)} \mathrm{d} \tau
$$

An incremental form of this expression is obtained by considering the substitutions

$$
\left\{\begin{array}{l}
\tau=t_{0}+s \\
t=t_{0}+\Delta t
\end{array} \Rightarrow \sigma=\sigma_{0} \mathrm{e}^{-\beta \Delta t}+\int_{0}^{\Delta t} E \dot{\varepsilon} \mathrm{e}^{-\beta(\Delta t-s)} \mathrm{d} s\right.
$$

where $\beta=E / \eta$ represents the relaxation modulus and $0 \leqslant s \leqslant \Delta t$ is the time variable in the time step $\Delta t$.

The fundamental difference between the models represented in Figures 1 and 2 is that in the first case (Figure 1) the viscosity affects only the plastic part of the deformation, while in the second case (Figure 2) the viscosity affects the total deformation. In what concerns the use of the Duvaut-Lions model and the proposed model, in the context of displacement-based finite element formulations (strain driven constitutive law), other important differences are:

Duvaut-Lions model

Requires an elasto-plastic integration procedure, in which the initial stress does not play a role, since it may be outside the yield surface. Usually, an Euler backward interpolation is used.

In the case of hardening or softening an evolution scheme for the hardening parameters must be postulated [4].

Only one new parameter must be given: the fluidity parameter.

\section{Proposed model}

Any integration scheme may be used in the elasto-plastic component of the model, since the elasto-plastic and the visco-elastic problems are completely independent at the local level. The same holds, obviously, for the visco-elastic component. In the present work different integration schemes are used for both components.

Nothing new must be postulated in what concerns the elasto-plastic law, since nothing is modified, in relation to the inviscid case.

Three new parameters are necessary, if the visco-elastic model is linear and isotropic: an Young modulus, a Poisson's coefficient and a viscosity parameter. In the described examples an almost purely viscous model is considered, since very high values of the Young modulus are used. 
2.2.1. Time integration algorithm. The time integration of the constitutive equation of the Maxwell model is performed using an indirect approximation of the strain. The algorithm considers visco-elastic linearity in the time step (constant values of $E$ and $\eta$ ), is implicit and uses a mid-point linear strain interpolation in the time step. This algorithm belongs to the family of the relaxation methods defined by Argyris et al. [7] and follows a pattern, which is similar to the one described by Barros et al. for the Kelvin-Voigt chain [8].

Denoting by $\varepsilon_{a}$ and $\varepsilon_{b}$ the strains at the beginning and at the end of the time step $\Delta t$, respectively, the strain interpolation function takes the form

$$
\varepsilon(s)=\varepsilon_{a}+\frac{\varepsilon_{b}-\varepsilon_{a}}{\Delta t} s \Rightarrow \dot{\varepsilon}=\frac{\varepsilon_{b}-\varepsilon_{a}}{\Delta t}=\frac{\Delta \varepsilon}{\Delta t}
$$

Substituting this expression in (4), the incremental stress-strain relation is obtained

$$
\begin{aligned}
\sigma & =\sigma_{0} \mathrm{e}^{-\beta \Delta t}+\frac{1-\mathrm{e}^{-\beta \Delta t}}{\beta \Delta t} E \Delta \varepsilon \\
& \Rightarrow \Delta \sigma=\sigma-\sigma_{0}=-\sigma_{0}\left(1-\mathrm{e}^{-\beta \Delta t}\right)+\frac{1-\mathrm{e}^{-\beta \Delta t}}{\beta \Delta t} E \Delta \varepsilon
\end{aligned}
$$

A three-dimensional generalization of Equation (6) is easily performed, if a constant Poisson's coefficient $v$ is used. Defining the matrix

$$
\boldsymbol{v}=\left[\begin{array}{cccccc}
1-v & v & v & 0 & 0 & 0 \\
v & 1-v & v & 0 & 0 & 0 \\
v & v & 1-v & 0 & 0 & 0 \\
0 & 0 & 0 & 1-2 v & 0 & 0 \\
0 & 0 & 0 & 0 & 1-2 v & 0 \\
0 & 0 & 0 & 0 & 0 & 1-2 v
\end{array}\right] \frac{1}{(1+v)(1-2 v)}
$$

the tensorial three-dimensional equivalent of $\Delta \sigma$ takes the form

$$
\Delta \boldsymbol{\sigma}=-\left(1-\mathrm{e}^{-\beta \Delta t}\right) \boldsymbol{\sigma}_{0}+\frac{1-\mathrm{e}^{-\beta \Delta t}}{\beta \Delta t} E \boldsymbol{v} \Delta \boldsymbol{\varepsilon}
$$

$\boldsymbol{\sigma}$ and $\varepsilon$ are vectors containing the elements of the stress and strain tensors, respectively. The visco-elastic material stiffness is then

$$
\frac{\mathrm{d} \Delta \boldsymbol{\sigma}}{\mathrm{d} \Delta \boldsymbol{\varepsilon}}=E \frac{1-\mathrm{e}^{-\beta \Delta t}}{\beta \Delta t} \boldsymbol{v}
$$

2.2.2. Stability analysis. In localization analysis it is important to use numerically stable time integration algorithms, since softening causes sudden deformations (localization in time) concentrated in narrow zones (space localization), which lead to larger deformation increments, even when small time steps are used. 


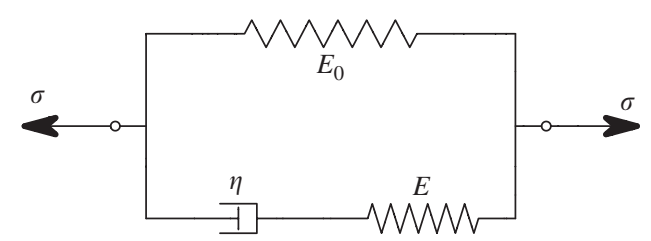

Figure 3. Rheological model used in the analysis of the numerical stability.

As it is not easy to include plasticity in the stability analysis of time-integration algorithms for visco-elasticity, which use an indirect approximation of the inelastic strain, the stability analysis of the time integration algorithm for the visco-elastic model component is performed considering the Maxwell element in conjunction with elastic behaviour. In this sense, the model presented in Figure 3 is used to investigate the stability and oscillation properties of the algorithm described in the previous section 2.2.1.

The stability of time integration algorithms is usually investigated by analyzing the decay properties of the numerical approximation to the homogeneous part of the differential equation which describes the problem. In the case of the model presented in Figure 3, its constitutive law may be described by the expression

$$
\dot{\varepsilon}=\left(\dot{\sigma}-E_{0} \dot{\varepsilon}\right) \frac{1}{E}+\left(\sigma-E_{0} \varepsilon\right) \frac{1}{\eta} \Rightarrow \sigma+\frac{\eta}{E} \dot{\sigma}=E_{0} \varepsilon+\eta \frac{E+E_{0}}{E} \dot{\varepsilon}
$$

which is obtained by adding the rate of deformation of the elements $E$ and $\eta$. $\varepsilon$ represents the total strain.

Following the argumentation presented by Argyris et al. [7], in a relaxation approach, as the one considered here, it suffices to analyse the local stability in a creep problem. Although the extension of this conclusion to softening plasticity is not straightforward, the creep of the model under constant stress is considered here.

Under these conditions, it is easy to verify that the homogeneous solution may be described by the strain in the spring $E$. In fact, the differential equation, which relates the stress with the strain in that spring, $\varepsilon_{s}$, may be obtained from Equation (10), taking the form

$$
\left\{\begin{array}{l}
\varepsilon=\frac{1}{E_{0}}\left(\sigma-E \varepsilon_{s}\right) \\
\dot{\varepsilon}=\frac{1}{E_{0}}\left(\dot{\sigma}-E \dot{\varepsilon}_{s}\right)
\end{array} \Rightarrow E \varepsilon_{s}+\eta \frac{E+E_{0}}{E_{0}} \dot{\varepsilon}_{s}=\frac{\eta}{E_{0}} \dot{\sigma}\right.
$$

It is obvious that this equation becomes homogeneous, if the stress $\sigma$ is constant.

In order to get an analytical description of the numerical approximation to the homogeneous part of the solution, a relation between the approximated variable - the strain $\varepsilon$ - and $\varepsilon_{s}$ is needed. This relation may be obtained in the same way as Equation (10), being given by

$$
\dot{\varepsilon}=\dot{\varepsilon}_{s}+\frac{E}{\eta} \varepsilon_{s}
$$


This equation may be integrated using the same technique as previously for Equation (4), yielding in incremental form

$$
\varepsilon_{s}=\varepsilon_{s 0} \mathrm{e}^{-\beta \Delta t}+\int_{0}^{\Delta t} \dot{\varepsilon} \mathrm{e}^{-\beta(\Delta t-s)} \mathrm{d} s
$$

where $\varepsilon_{s 0}$ represents the value of $\varepsilon_{s}$ for $s=0$ and $\beta=E / \eta$.

From Equations (5) and (11) the strain rate is obtained

$$
\left\{\begin{array}{l}
\dot{\varepsilon}=\frac{\varepsilon_{b}-\varepsilon_{a}}{\Delta t} \\
\varepsilon=\frac{1}{E_{0}} \sigma-\frac{E}{E_{0}} \varepsilon_{s}
\end{array} \Rightarrow \dot{\varepsilon}=-\frac{E}{E_{0}} \frac{\varepsilon_{s b}-\varepsilon_{s a}}{\Delta t}\right.
$$

representing $\varepsilon_{s a}$ and $\varepsilon_{s b}$ the values of $\varepsilon_{s}$ at the beginning and at the end of the time step, respectively. Substituting this equation in (13) and integrating, we get $\left(\varepsilon_{s}=\varepsilon_{s b}\right.$ and $\left.\varepsilon_{s 0}=\varepsilon_{s a}\right)$

$$
\varepsilon_{s b}=\varepsilon_{s a} \mathrm{e}^{-\beta \Delta t}+\frac{E}{E_{0}} \frac{\varepsilon_{s a}-\varepsilon_{s b}}{\Delta t} \frac{1}{\beta}\left(1-\mathrm{e}^{-\beta \Delta t}\right)
$$

Rearranging, we get

$$
\varepsilon_{s b}=A \varepsilon_{s a} \quad \text { with } \quad A=\frac{\mathrm{e}^{-\beta \Delta t}+\frac{E}{E_{0}} \frac{1-\mathrm{e}^{-\beta \Delta t}}{\beta \Delta t}}{1+\frac{E}{E_{0}} \frac{1-\mathrm{e}^{-\beta \Delta t}}{\beta \Delta t}}
$$

For a given starting value $\varepsilon_{s 1}$, the value of $\varepsilon_{s}$ after $n$ time steps, $\varepsilon_{s n}$, is then given by

$$
\varepsilon_{s n}=A^{n} \varepsilon_{s 1}
$$

$A$ represents the amplification function. If $-1 \leqslant|A| \leqslant 1$, the truncation errors are not amplified and the algorithm is numerically stable. Furthermore, if $A \geqslant 0$, the algorithm does not oscillate. It is easy to see that both of these conditions are satisfied for any value of $\Delta t$. This means that the algorithm is unconditionally stable and oscillation free.

In order to verify these conclusions, the analytical solution of Equation (10) for constant $\sigma$ (creep problem) is compared with the approximated solution obtained using the time integration defined by Equation (6). The integration of Equation (10) furnishes the solution $(\dot{\sigma}=0)$

$$
\varepsilon=\frac{\sigma}{E_{0}}+C \mathrm{e}^{-\lambda t} \quad \text { with } \quad \lambda=\frac{1}{\eta} \frac{E E_{0}}{E+E_{0}}
$$

where $C$ is a constant, which may be eliminated by considering the initial strain $\varepsilon=\sigma /\left(E+E_{0}\right)$ for $t=0$. With this condition Equation (18) yields

$$
\varepsilon(t)=\frac{\sigma}{E_{0}}\left(1-\frac{E}{E+E_{0}} \mathrm{e}^{-\lambda t}\right)
$$

This creep function may also be computed by means of the algorithm presented in Section 2.2.1. Using Equation (6), the relation between the stress increment $\Delta \sigma$ and the strain 


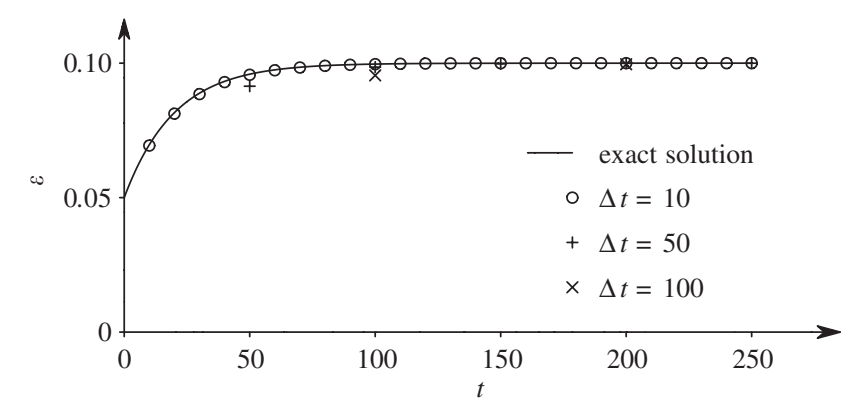

Figure 4. Exact versus approximate solutions for several time increments.

increment $\Delta \varepsilon$ is

$$
\Delta \sigma=E_{0} \Delta \varepsilon-\sigma_{0 m}\left(1-\mathrm{e}^{-\beta \Delta t}\right)+\frac{1-\mathrm{e}^{-\beta \Delta t}}{\beta \Delta t} E \Delta \varepsilon
$$

where $\sigma_{0 m}$ represents the stress in the Maxwell element. As $\sigma$ is constant, we have

$$
\Delta \sigma=0 \Rightarrow \Delta \varepsilon=\frac{\left(1-\mathrm{e}^{-\beta \Delta t}\right) \sigma_{0 m}}{E_{0}+\frac{1-\mathrm{e}^{-\beta \Delta t}}{\beta \Delta t} E}
$$

After computation of $\Delta \varepsilon$ the value of $\sigma_{0 m}$ is updated using Equation (6)

$$
\sigma_{m}=\sigma_{0 m} \mathrm{e}^{-\beta \Delta t}+\frac{1-\mathrm{e}^{-\beta \Delta t}}{\beta \Delta t} E \Delta \varepsilon \rightarrow \sigma_{0 m}=\sigma_{m}
$$

In Figure 4 the results obtained using the analytical solution (19) and the numerical approach with three different values of $\Delta t$ are presented. In this example the following values of the involved quantities were used: $E=E_{0}=100, \beta=0.1$ and $\sigma=10$. The initial value of $\sigma_{0 m}$ is $\sigma_{0 m}=E /\left(E+E_{0}\right) \sigma$.

The results confirm that, even for very large time steps, the algorithm is stable and free of oscillations. Furthermore, it can be verified that the degree of accuracy is very high.

In order to test the stability properties of the time integration algorithm in presence of plasticity, the creep behaviour of the complete model (Figure 2) was computed. The constitutive equations of the one-dimensional elasto-plastic model are

$$
\begin{aligned}
F & =\sigma-\sigma_{c}=0 \\
\dot{\varepsilon}^{\mathrm{p}} & =\dot{\lambda} \\
\sigma_{c} & =\sigma_{c 0}\left(1+\alpha \int \mathrm{d} \varepsilon^{\mathrm{p}}\right) \Rightarrow \dot{\sigma}_{c}=\sigma_{c 0} \alpha \dot{\varepsilon}^{\mathrm{p}}=\sigma_{c 0} \alpha \dot{\lambda} \\
\dot{\sigma} & =E_{0}\left(\dot{\varepsilon}-\dot{\varepsilon}^{\mathrm{p}}\right)=E_{0}(\dot{\varepsilon}-\dot{\lambda})
\end{aligned}
$$

where $\sigma_{c 0}$ and $\alpha$ are the initial yield stress and the hardening parameter, respectively. The rate equations are then integrated by backward Euler interpolation, yielding for the plastic 
multiplier $\Delta \lambda_{n+1}=\dot{\lambda}_{n+1} \Delta t$ the value

$$
\begin{gathered}
\left\{\begin{array}{c}
\sigma_{n+1}=\sigma_{n}+E_{0} \Delta \varepsilon-E_{0} \Delta \lambda_{n+1} \\
\sigma_{c n+1}=\sigma_{c n}+\sigma_{c 0} \alpha \Delta \lambda_{n+1}
\end{array}\right. \\
\sigma_{n+1}-\sigma_{c n+1}=0 \Rightarrow \Delta \lambda_{n+1}=\frac{\sigma_{n}-\sigma_{c n}+E_{0} \Delta \varepsilon}{E_{0}+\alpha \sigma_{c 0}}
\end{gathered}
$$

The stress in the elasto-plastic element and the yielding stress, after the strain increment $\Delta \varepsilon$ may then be computed by means of Equations (24). By adding the stresses in both elements (visco-elastic and elasto-plastic), the total stress increment caused by given strain and time increments $\Delta \varepsilon$ and $\Delta t$, respectively, may be computed, cf. Equations (22), (24) and (25)

$$
\Delta \sigma=-\frac{E_{0}\left(\sigma_{n}-\sigma_{c n}\right)}{E_{0}+\alpha \sigma_{c 0}}+\frac{E_{0} \alpha \sigma_{c 0}}{E_{0}+\alpha \sigma_{c 0}} \Delta \varepsilon-\sigma_{0 m}\left(1-\mathrm{e}^{-\beta \Delta t}\right)+\frac{1-\mathrm{e}^{-\beta \Delta t}}{\beta \Delta t} E_{0} \Delta \varepsilon
$$

As the global stress is constant, the strain increment after creep during the time $\Delta t$ is given by

$$
\Delta \sigma=0 \Rightarrow \Delta \varepsilon=\frac{\frac{E_{0}\left(\sigma_{n}-\sigma_{c n}\right)}{E_{0}+\alpha \sigma_{c 0}}+\sigma_{0 m}\left(1-\mathrm{e}^{-\beta \Delta t}\right)}{\frac{E_{0} \alpha \sigma_{c 0}}{E_{0}+\alpha \sigma_{c 0}}+\frac{1-\mathrm{e}^{-\beta \Delta t}}{\beta \Delta t} E_{0}}
$$

The stress in the Maxwell element is updated using Equation (22).

In Figure 5 the results of computations considering hardening, perfect plasticity, softening and no plasticity are depicted. Although the performance, in the softening case, is not as good as in the other cases, it is still acceptable, even with the large time increment considered (it is larger than the relaxation time of the Maxwell element). Nevertheless, and in opposition to the non-softening case, the performance in the global problem deteriorates, as a consequence of the strain concentration which results from localization.

However, the high stability of the visco-elastic time integration algorithm has a strong stabilizing effect on the global problem, which is reflected in the fact that, with viscosity, much higher time steps are possible, than with the inviscid elasto-plastic material law, as referred later.

\subsection{Elasto-plastic element}

The integration scheme for the elasto-plastic component of the constitutive law does not need to be the same as for the visco-elastic Maxwell element. In the examples included in this report the backward linear Euler interpolation was chosen, since it leads to a simple implementation and to a relatively stable algorithm (in the hardening case it is unconditionally stable). Furthermore, as in the inviscid reference solutions an algorithm must be used, in which the initial stress does not play a role, this option facilitates the comparison between the results, since the inviscid solution is computed using the same interpolation scheme, both in the reference and proposed solutions. This interpolation scheme leads to a radial return of the deviatoric component of the stress tensor, when shapes of revolution around the hydrostatic axis are used for the yield and plastic potential functions. This is the case when those functions are expressed in terms of the two first invariants of the stress tensor. 


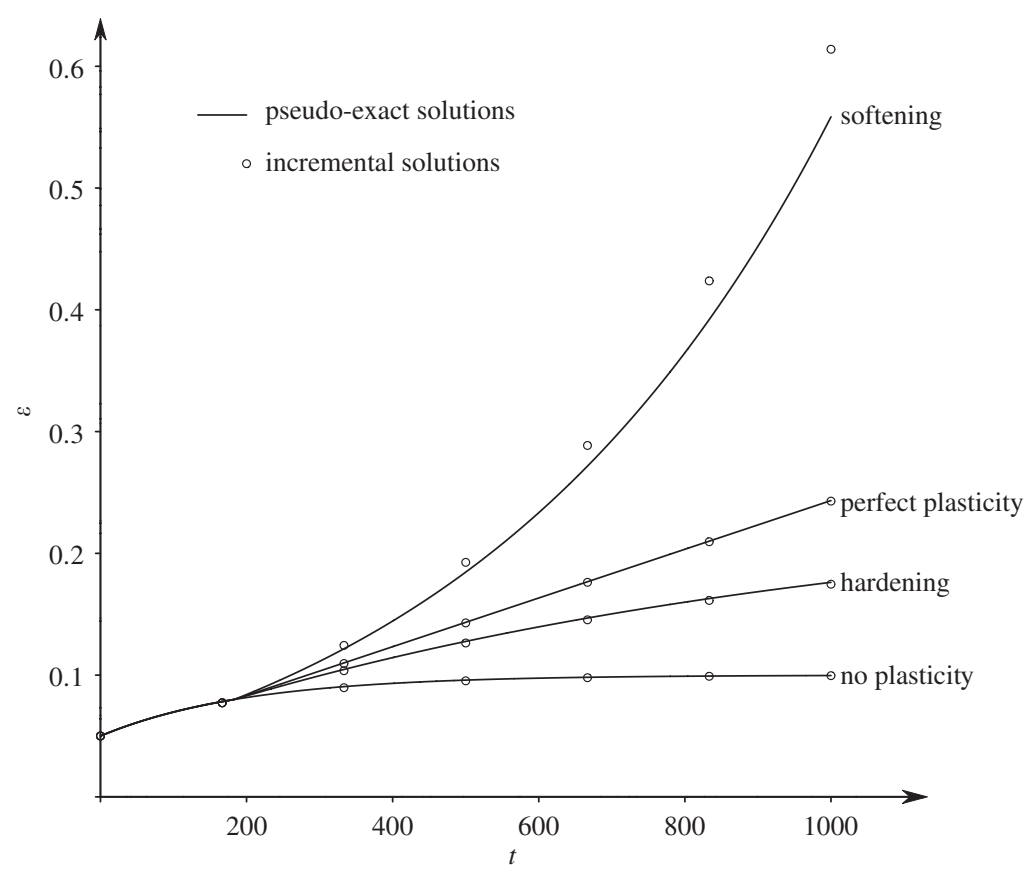

Figure 5. Numerical test of the used time-integration algorithm for visco-elasticity with plasticity. Rheological parameters: $E=E_{0}=100, \beta=0.01, \sigma_{c 0}=8$ (initial yield stress), $\alpha=1.75$ (hardening case) and $\alpha=-1.75$ (softening case). Time step: $\Delta t=166.7$. Creep stress: $\sigma=10$.

\section{EXAMPLES}

\subsection{Computation of reference solutions}

As referred in Section 1, the visco-plastic constitutive laws of Perzyna and Duvaut-Lions are the most used models to introduce viscosity in inviscid elasto-plastic material laws. The second one is used here to compute reference solutions for comparison with the results furnished by the presented overlay model.

The constitutive law of the Duvaut-Lions model may be described by the expression [3]

$$
\dot{\boldsymbol{\varepsilon}}_{v p}=\frac{1}{\tau} \mathbf{D}^{-1}(\boldsymbol{\sigma}-\overline{\boldsymbol{\sigma}})
$$

where $\tau$ represents the relaxation time (in the one-dimensional model, cf. Figure 1, this quantity is given by $\tau=\eta / E$ ), $\mathbf{D}$ the elastic material stiffness, $\boldsymbol{\sigma}$ the stress tensor and $\overline{\boldsymbol{\sigma}}$ the inviscid stress. In presence of hardening/softening, an evolution equation for the hardening parameter must be postulated. In the present contribution, the following law is used [4]

$$
\dot{q}=-\frac{1}{\tau}(q-\bar{q})
$$

where $q$ represents the actual value of the hardening parameter and $\bar{q}$ the inviscid solution, i.e. the value this parameter would take after relaxation. 
The time integration of Equations (28) and (29) may be performed in closed form [4], yielding the following expression for the stress at the end of the time increment $\Delta t$

$$
\boldsymbol{\sigma}_{n+1}=\boldsymbol{\sigma}_{n} \mathrm{e}^{-\beta \Delta t}+\overline{\boldsymbol{\sigma}}_{n+1}\left(1-\mathrm{e}^{-\beta \Delta t}\right)+\frac{1-\mathrm{e}^{-\beta \Delta t}}{\beta \Delta t} \mathbf{D} \Delta \boldsymbol{\varepsilon}
$$

given the strain increment $\Delta \boldsymbol{\varepsilon}$ and the inviscid elasto-plastic solution $\overline{\boldsymbol{\sigma}}_{n+1} \cdot \beta=1 / \tau$ represents the relaxation modulus. This equation is valid only in the case of a linear relation between the stress and the elastic strain, represented by the constant elastic material stiffness D. However, in the second of the presented examples - the Cam-Clay material model, cf. Section 3.3-the elastic stiffness is not constant. Therefore, Equation (30) has been used in the modified version represented by the expression

$$
\boldsymbol{\sigma}_{n+1}=\boldsymbol{\sigma}_{n} \mathrm{e}^{-\beta \Delta t}+\overline{\boldsymbol{\sigma}}_{n+1}\left(1-\mathrm{e}^{-\beta \Delta t}\right)+\frac{1-\mathrm{e}^{-\beta \Delta t}}{\beta \Delta t}\left(\boldsymbol{\sigma}_{n+1}^{\mathrm{tr}}-\boldsymbol{\sigma}_{n}\right)
$$

where $\mathbf{D} \Delta \boldsymbol{\varepsilon}$ is substituted by $\boldsymbol{\sigma}_{n+1}^{\mathrm{tr}}-\boldsymbol{\sigma}_{n} . \boldsymbol{\sigma}_{n+1}^{\mathrm{tr}}$ represents the trial stress introduced by the strain increment $\Delta \boldsymbol{\varepsilon}$. There is, however, a more accurate solution, obtained by the integration of Equation (28) in the special case of the Cam-Clay model, which, for the case of constant shear modulus $G$, takes the form [9]

$$
\begin{aligned}
& \sigma_{n+1}=\sigma_{n} \mathrm{e}^{-\beta \Delta t}+\bar{\sigma}_{n+1}\left(1-\mathrm{e}^{-\beta \Delta t}\right)+\sigma_{n} \frac{1}{\Delta \varepsilon_{v}-k \beta \Delta t}\left(\mathrm{e}^{-\frac{\Delta \varepsilon_{v}}{k}}-\mathrm{e}^{-\beta \Delta t}\right) \Delta \varepsilon_{v} \\
& \boldsymbol{\sigma}_{n+1}^{\prime}=\boldsymbol{\sigma}_{n}^{\prime} \mathrm{e}^{-\beta \Delta t}+\overline{\boldsymbol{\sigma}}_{n+1}^{\prime}\left(1-\mathrm{e}^{-\beta \Delta t}\right)+2 G \frac{1-\mathrm{e}^{-\beta \Delta t}}{\beta \Delta t} \Delta \boldsymbol{\varepsilon}^{\prime}
\end{aligned}
$$

respectively for the mean stress $\sigma$ and for the deviatoric stress tensor $\boldsymbol{\sigma}^{\prime} . k$ is a rheological parameter (cf. Section 3.3). The results obtained using Equation (31) or (32) are, however, practically the same. In both cases, as a consequence of the non-linear elastic behaviour, the relaxation modulus $\beta$ is prescribed and not the viscosity $\eta(\beta=E / \eta$ in the one-dimensional case).

By integration of Equation (29), the hardening parameter at the end of the time increment $\Delta t$ is given by [4]

$$
q_{n+1}=q_{n} \mathrm{e}^{-\beta \Delta t}+\left(1-\mathrm{e}^{-\beta \Delta t}\right) \bar{q}_{n+1}
$$

where $\bar{q}_{n+1}$ is computed by integration of the inviscid rate equations.

The material stiffness may be obtained by derivation of Equation (31) or (32), yielding in the case of Equation (31)

$$
\begin{aligned}
\mathbf{D}_{\text {vep }} & =\frac{\partial \boldsymbol{\sigma}_{n+1}}{\partial \Delta \boldsymbol{\varepsilon}}=\left(1-\mathrm{e}^{-\beta \Delta t}\right) \frac{\partial \overline{\boldsymbol{\sigma}}_{n+1}}{\partial \Delta \boldsymbol{\varepsilon}}+\frac{1-\mathrm{e}^{-\beta \Delta t}}{\beta \Delta t} \frac{\partial \boldsymbol{\sigma}_{n+1}^{\mathrm{tr}}}{\partial \Delta \boldsymbol{\varepsilon}} \\
& =\left(1-\mathrm{e}^{-\beta \Delta t}\right) \mathbf{D}_{\mathrm{ep}}+\frac{1-\mathrm{e}^{-\beta \Delta t}}{\beta \Delta t} \mathbf{D}
\end{aligned}
$$

where $\mathbf{D}_{\mathrm{ep}}=\partial \overline{\boldsymbol{\sigma}}_{n+1} / \partial \Delta \boldsymbol{\varepsilon}$ represents the inviscid elasto-plastic material stiffness. In the first of the examples described in the present communication - the von Mises material — an algorithmic 
material stiffness has been computed, while in the second one-the Cam-Clay model - the classical expression for associative plasticity is used

$$
\mathbf{D}_{\mathrm{ep}}=\mathbf{D}-\frac{\mathbf{D a a}^{\mathrm{T}} \mathbf{D}}{\mathbf{a}^{\mathrm{T}} \mathbf{D a}+A}
$$

where a defines the direction of the plastic flow (normal to the yield surface) and $A$ reflects the influence of the hardening rule. An algorithmic version of $\mathbf{D}_{\text {ep }}$ did not bring advantages in this case, since it had to be computed numerically, as a consequence of the non-linear character of the inviscid rate equations, which leads to an iterative computation of the elasto-plastic stresses and hardening parameter, as referred in Section 3.3.

\subsection{Von Mises material}

In order to test the regularization capabilities of the proposed procedure in a material which displays slip-driven softening, the most simple material law for ductile materials - the von Mises material—was implemented in a FE-code for non-linear analysis [10].

The yielding function of the von Mises material may be defined by the expression

$$
F=\sqrt{3} \tau-\sigma_{c}=0 \quad \text { with } \quad \tau=\sqrt{J_{2}^{\prime}} \quad \text { and } \quad J_{2}^{\prime}=\frac{1}{2} \sigma_{i j}^{\prime} \sigma_{i j}^{\prime}=\frac{1}{2} \sigma_{i j} \sigma_{i j}-\frac{1}{6} I_{1}^{2}
$$

where $\sigma_{c}$ is the hardening parameter, $\sigma_{i j}$ and $\sigma_{i j}^{\prime}$ are the elements of the stress tensor and stress deviation tensor, respectively, and $I_{1}$ is the first invariant of the stress tensor.

The flow rule is associative. The hardening rule is defined by the expression

$$
\sigma_{c}=\sigma_{c 0}\left(1+\alpha \int \mathrm{d} \varepsilon^{\mathrm{p}}\right) \quad \text { with } \quad \mathrm{d} \varepsilon^{\mathrm{p}}=\sqrt{\frac{1}{2} \mathrm{~d} \varepsilon_{i j}^{\mathrm{p}} \mathrm{d} \varepsilon_{i j}^{\mathrm{p}}}
$$

where $\sigma_{c 0}$ is the initial value of the hardening parameter $\sigma_{c}$ (yield stress) and $\mathrm{d} \varepsilon_{i j}^{\mathrm{p}}$ are the components of the incremental plastic strain tensor. A negative value of the parameter $\alpha$ leads to softening.

The numerical integration of the rate equations, performed via backward linear interpolation, follows the scheme

$$
\Delta \boldsymbol{\sigma}=\dot{\boldsymbol{\sigma}}_{n+1} \Delta t \quad \text { and } \quad \Delta \sigma_{c}=\dot{\sigma}_{c n+1} \Delta t
$$

where the index ' $n+1$ ' denotes the end of the loading step. This means that all the parameters included in the rate equations take the (unknown) values corresponding to the new stress state.

The development of the expressions corresponding to the numerical integration of the rate equations, performed via backward linear interpolation (Equation (38)), following a standard pattern, is not presented here. The resulting expressions allow the computation of the new stress and the corresponding material stiffness, given the starting stress tensor $\boldsymbol{\sigma}_{n}$ and the strain-increment tensor $\Delta \varepsilon$, which follows the steps:

- identification of the plastic deformation 
- computation of the new stresses and hardening parameter using the expressions obtained via backward linear interpolation

$$
\begin{gathered}
\boldsymbol{\sigma}_{n+1}=\left(\sigma_{n}+K \Delta \varepsilon_{v}\right) i+\frac{1}{\tau_{\mathrm{tr}}}\left(\sigma_{c n}+\frac{\sqrt{3}}{2} \sigma_{c 0} \alpha \Delta \lambda_{n+1}\right) \boldsymbol{\sigma}_{\mathrm{tr}}^{\prime} \\
\sigma_{c n+1}=\sigma_{c n}+\frac{\sqrt{3}}{2} \sigma_{c 0} \alpha \Delta \lambda_{n+1} \quad \text { with } \quad \Delta \lambda_{n+1}=\frac{\sqrt{3} \tau_{\mathrm{tr}}-\sigma_{c n}}{\sigma_{c 0} \alpha \frac{\sqrt{3}}{2}+3 G}
\end{gathered}
$$

where $K$ and $G$ represent the bulk and shear moduli, respectively, $\tau_{\text {tr }}$ is the square root of the second invariant of the deviatoric trial stress $\boldsymbol{\sigma}_{\mathrm{tr}}^{\prime}$ and $\mathbf{i}=\left[\begin{array}{llllll}1 & 1 & 1 & 0 & 0 & 0\end{array}\right]^{T} . \sigma_{n}$ and $\sigma_{c n}$ are the mean and yielding stresses, respectively, at the beginning of the loading step.

- computation of the algorithmic elasto-plastic material stiffness for the new stress tensor, using the derivative of Equation (39)

$$
\frac{\partial \boldsymbol{\sigma}_{n+1}}{\partial \Delta \boldsymbol{\varepsilon}}=K \mathbf{B}+\left[\sigma_{c n}\left(1-\frac{\sqrt{3} \sigma_{c 0} \alpha}{\sqrt{3} \sigma_{c 0} \alpha+6 G}\right)\left(\frac{1}{\tau_{\mathrm{tr}}} \mathbf{I}-\frac{1}{2 \tau_{\mathrm{tr}}^{3}} \boldsymbol{\sigma}_{\mathrm{tr}}^{\prime} \boldsymbol{\sigma}_{\mathrm{tr}}^{\prime T}\right)+\frac{3 \sigma_{c 0} \alpha}{\sqrt{3} \sigma_{c 0} \alpha+6 G} \mathbf{I}\right] 2 G \mathbf{C}
$$

where $\mathbf{I}$ is the sixth-rank identity matrix, while $\mathbf{B}$ and $\mathbf{C}$ are defined as

$$
\mathbf{B}=\left[\begin{array}{llllll}
1 & 1 & 1 & 0 & 0 & 0 \\
1 & 1 & 1 & 0 & 0 & 0 \\
1 & 1 & 1 & 0 & 0 & 0 \\
0 & 0 & 0 & 0 & 0 & 0 \\
0 & 0 & 0 & 0 & 0 & 0 \\
0 & 0 & 0 & 0 & 0 & 0
\end{array}\right] \quad \mathbf{C}=\left[\begin{array}{cccccc}
\frac{2}{3} & -\frac{1}{3} & -\frac{1}{3} & 0 & 0 & 0 \\
-\frac{1}{3} & \frac{2}{3} & -\frac{1}{3} & 0 & 0 & 0 \\
-\frac{1}{3} & -\frac{1}{3} & \frac{2}{3} & 0 & 0 & 0 \\
0 & 0 & 0 & 1 & 0 & 0 \\
0 & 0 & 0 & 0 & 1 & 0 \\
0 & 0 & 0 & 0 & 0 & 1
\end{array}\right]
$$

As a numerical application to the von Mises material, the process of formation of a shear band in a specimen of this material is simulated. The specimen has the dimensions $300 \times$ $100 \times 10 \mathrm{~mm}^{3}$ with a non prismatic shape for stress concentration (Figure 6). Pentahedric elements with two integration points are used. A displacement of $0.04167 \mathrm{~mm} / \mathrm{s}$ is imposed in the upper face.

For the elasto-plastic law an yielding stress of $235 \mathrm{MPa}$, an Young modulus of $206 \mathrm{GPa}$ and a Poisson's coefficient of 0.3 are used. The used hardening parameter $\alpha$ has the value $\alpha=-6.0$. With this value of $\alpha$ a pronounced softening behaviour is obtained. As a consequence of this, the global stiffness matrix becomes singular, even for small loading increments and, therefore, a very large number of loading steps is necessary, although the used algorithms are unconditionally stable in case of hardening plasticity: if a positive value of $\alpha$ is used (hardening plasticity), the algorithm remains numerically stable, even when the loading is applied in only one step. The introduction of viscosity allows for much larger loading steps, 


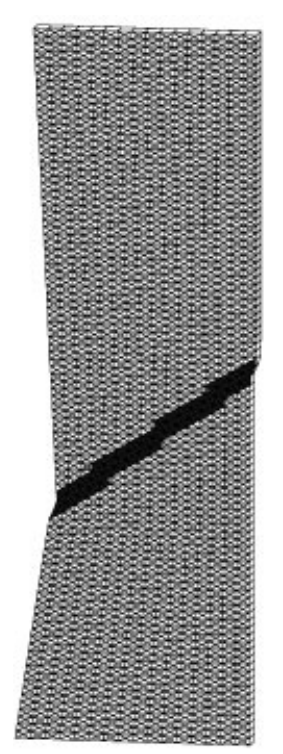

$t=72 s$

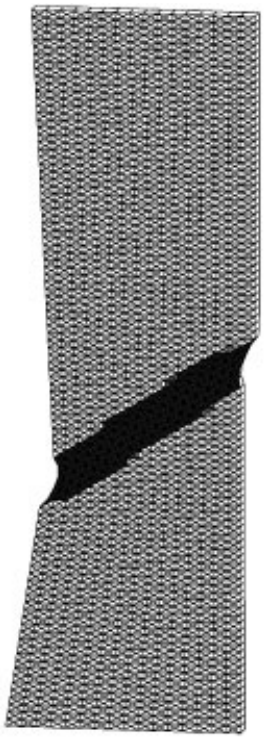

$t=144 \mathrm{~s}$

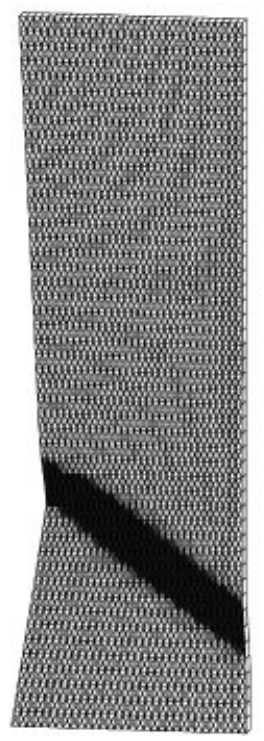

$t=72 \mathrm{~s}$

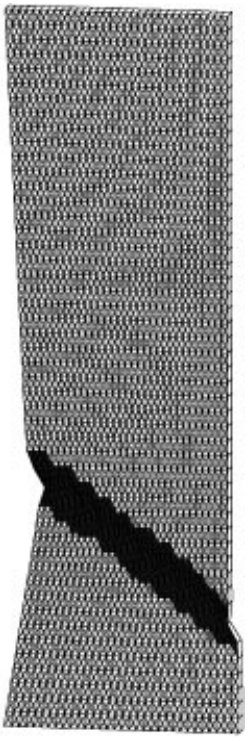

$t=144 s$

mesh 1

mesh 2

Figure 6. Shear bands in a von Mises specimen using two different meshes: computation with low viscosity using the Duvaut-Lions model $\left(\beta=150 \mathrm{~s}^{-1}\right)$.

which is of crucial importance in this kind of problems, since very refined meshes must be used, in order to capture the formation of shear bands. In the examples presented here, loading steps of $1 \mathrm{~s}(0.04167 \mathrm{~mm})$ were used.

For these reasons, the regularization capabilities of the proposed method are investigated by comparing a less regularized solution with a more regularized one. The less regularized solution is computed using both the Duvaut-Lions (Figure 6) and the overlay model (Figure 7). In order to have an almost purely viscous behaviour in the Maxwell element of the overlay model, an Young modulus of $20600 \mathrm{GPa}$ was considered in this element. For the Poisson's coefficient of the visco-elastic model the same value as in the elasto-plastic law $(v=0.3)$ is used. The less regularized solutions display the mesh sensitivity presented in Figures 6 and 7 , which is characterized by different directions of the shear bands in the two meshes.

Figure 8 shows the results obtained when the viscosity is introduced by means of the Duvaut-Lions model with a value of $\beta=15 \mathrm{~s}^{-1}$ for the relaxation modulus. It can be observed that the results obtained are more similar, with shear bands with the same directions in both meshes.

Practically the same results are obtained when the overlay model is used to introduce viscosity (Figure 9) in the constitutive law, as can be seen by comparing Figures 8 and 9. In both the Duvaut-Lions and the overlay models the used value for the viscosity is about 2.3 times the minimum value to force the final shear band in mesh 2 to take the same direction as in mesh 1. 


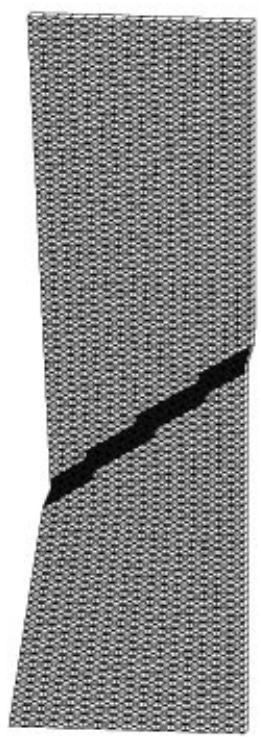

$t=72 \mathrm{~s}$

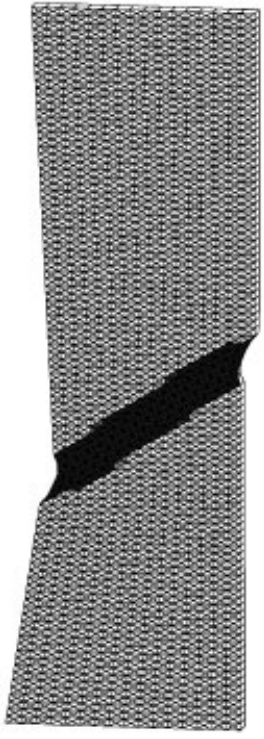

$t=144 s$

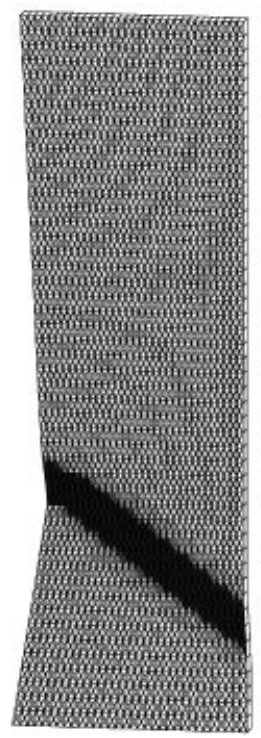

$t=72 \mathrm{~s}$

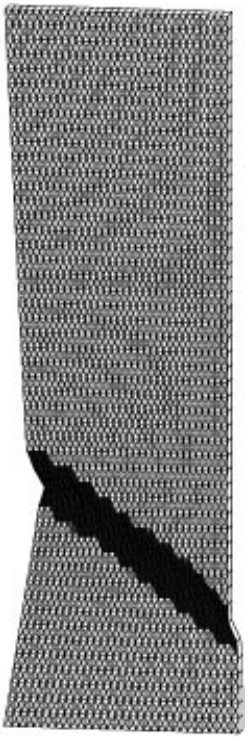

$t=144 s$

mesh 1

mesh 2

Figure 7. Shear bands in a von Mises specimen using two different meshes: computation with low viscosity using the overlay model $\left(\beta=15000 \mathrm{~s}^{-1}\right)$.

Since no inertial forces are taken in account, it is obvious that other values of $\beta$ would lead to the same results, provided that values of the time $t$ are considered, such that $\beta t$ remains constant.

\subsection{Cam-Clay material}

The Cam-Clay model displays decohesion driven softening, when the isotropic compressive stress takes a smaller value than the so-called critical stress. In the present contribution only effective stresses are considered. Although it is usual in Soil Mechanics to consider the compressive stresses as positive, here they are considered as negative, since this constitutive law has been implemented in a Finite Element program system which use this sign convention [10].

In Figure 10 the volumetric elasto-plastic material behaviour is presented. The rheological parameter $\lambda$ defines the behaviour in a normal consolidation process, which in a semilogarithmic referential may be represented as a straight line. The parameter $k$ defines the unload-reload behaviour and allows for the identification of the plastic deformation component.

Considering a load increment of the isotropic compressive stress $P_{0}$, from $P_{01}$ to $P_{02}$, the corresponding increments of volumetric elastic and plastic deformation $\Delta \varepsilon_{v}^{\mathrm{e}}$ and $\Delta \varepsilon_{v}^{\mathrm{p}}$ are 

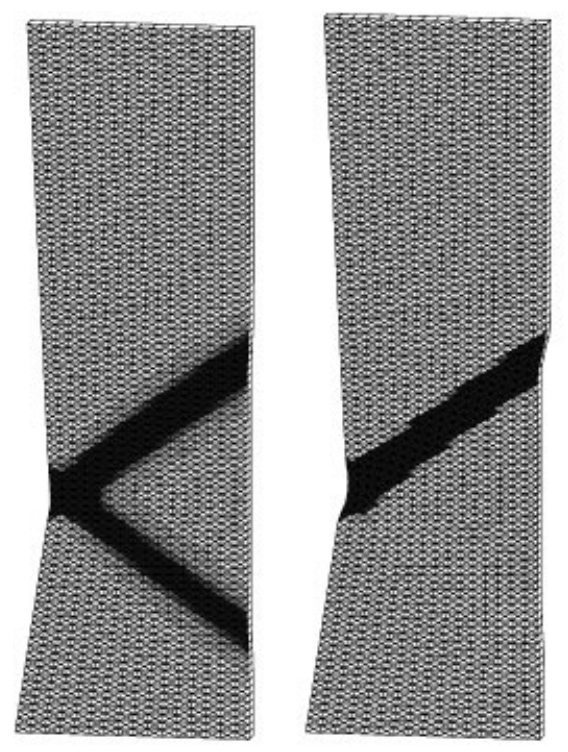

$$
t=72 \mathrm{~s} \underset{\text { mesh 1 }}{ } t=144 \mathrm{~s}
$$
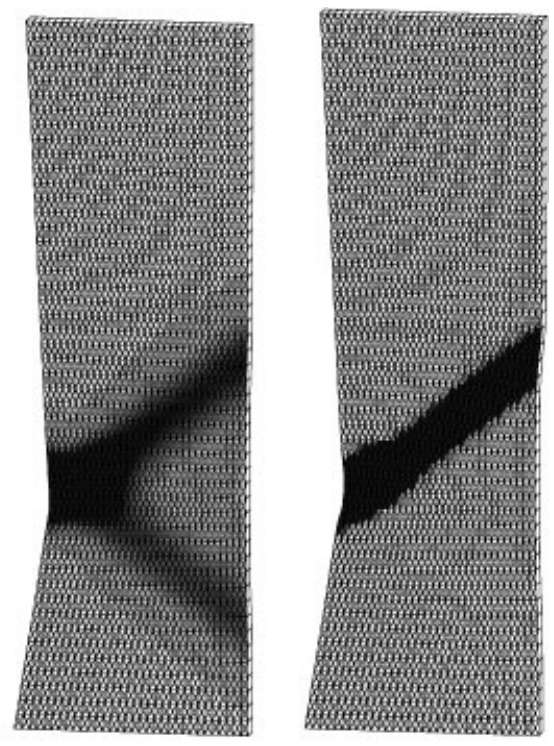

$t=72 s$

$t=144 \mathrm{~s}$

Figure 8. Shear bands in a von Mises specimen using two different meshes and the Duvaut-Lions model $\left(\beta=15 \mathrm{~s}^{-1}\right)$.

given by

$$
\Delta \varepsilon_{v}^{\mathrm{e}}=-k \ln \left(\frac{P_{02}}{P_{01}}\right) \quad \text { and } \quad \Delta \varepsilon_{v}^{\mathrm{p}}=-(\lambda-k) \ln \left(\frac{P_{02}}{P_{01}}\right)
$$

In the Cam-Clay elasto-plastic model $P_{0}$ is the hardening parameter playing the role of a cohesion parameter. The yield locus has the shape of an ellipsoid of revolution around the hydrostatic axis. The relation between the transversal and longitudinal axis is defined by the parameter $M$. The plastic potential is associative.

The shear modulus $G$ or the Poisson's coefficient $v$ complete the set of parameters necessary for the description of the elasto-plastic material behaviour of the Cam-Clay model.

As a consequence of the non-linear character of the elastic deformation, flow rule and hardening rule and of the use of a backward algorithm, the numerical integration of the rate equations leads to a non-linear system of three equations, which is solved by means of a Newton-Raphson iterative procedure [9].

In the present work, material parameters taken from Reference [11] have been used: $\lambda=$ $0.042, k=0.015, M=0.882, P_{0}=138 \mathrm{kPa}$ and $v=0.3$. With this value of $P_{0}$ and a lateral pressure $p=15 \mathrm{kPa}$, a pronounced softening behaviour is obtained, as indicated in Figure 11 . In this Figure, the visco-elasto-plastic stress-strain relation, obtained with the values of the 

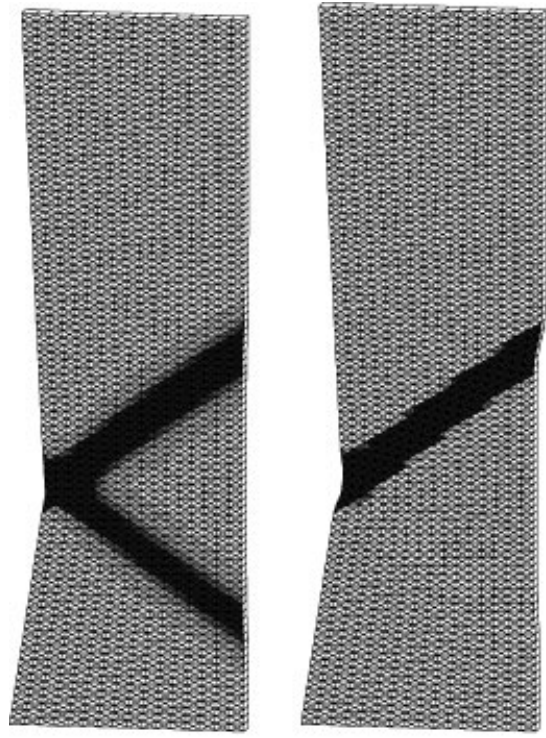

$t=72 s$

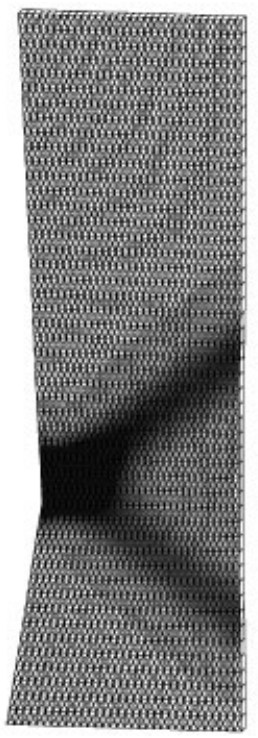

$t=72 \mathrm{~s}$

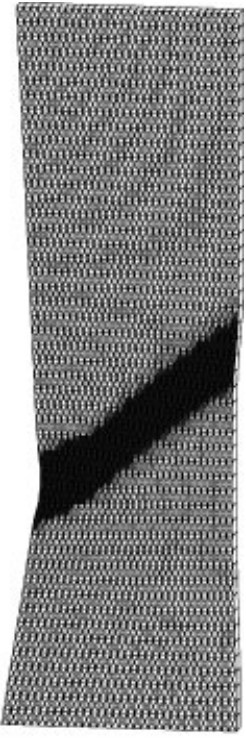

$t=144 \mathrm{~s}$

mesh 1

mesh 2

Figure 9. Shear bands in a von Mises specimen using two different meshes and the overlay model $\left(\beta=1500 \mathrm{~s}^{-1}\right)$.

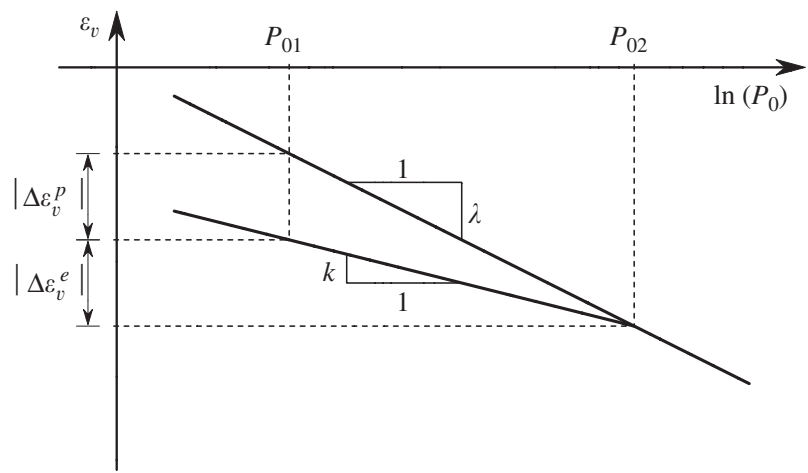

Figure 10. Elastic and plastic parts of a volumetric strain increment.

confinement pressure, relaxation modulus $\beta$ and strain rate used in the example of regularization described in this section, are also presented.

In the case of the overlay model, an almost purely viscous behaviour in the visco-elastic model is achieved by considering a high value for the Young modulus $E=50 \times 10^{6} \mathrm{kPa}$, 


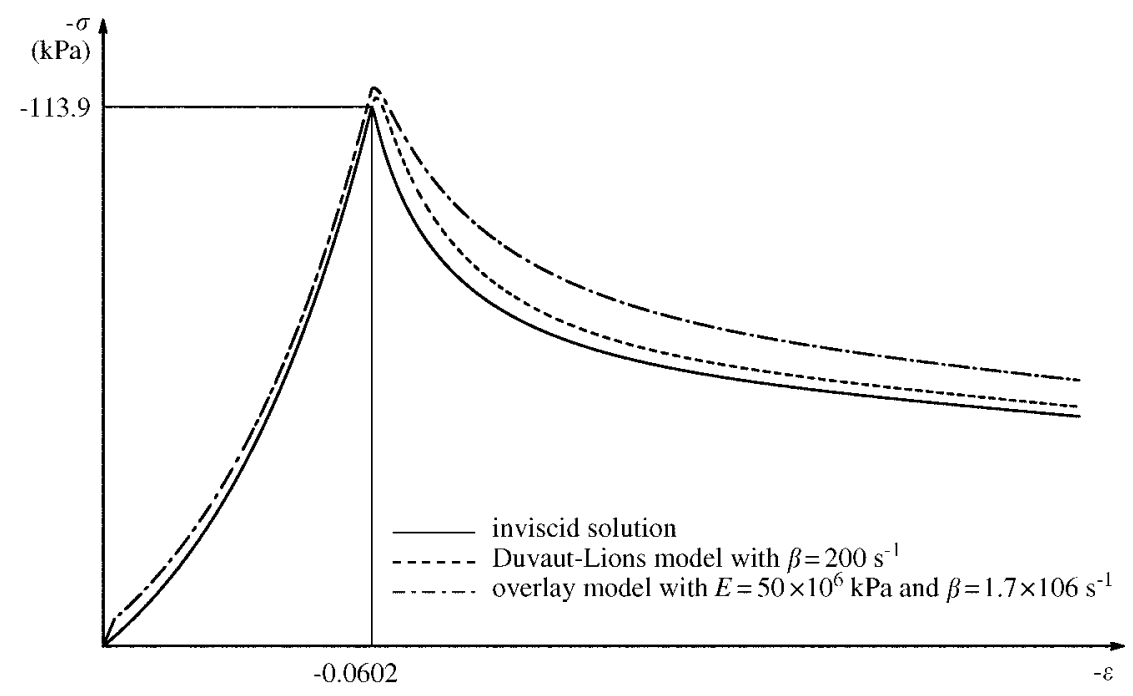

Figure 11. One-dimensional stress-strain relation of the used Cam-Clay model, with a confinement pressure of $15 \mathrm{MPa}$ and a deformation rate of $0.125 \mathrm{~s}^{-1}$.
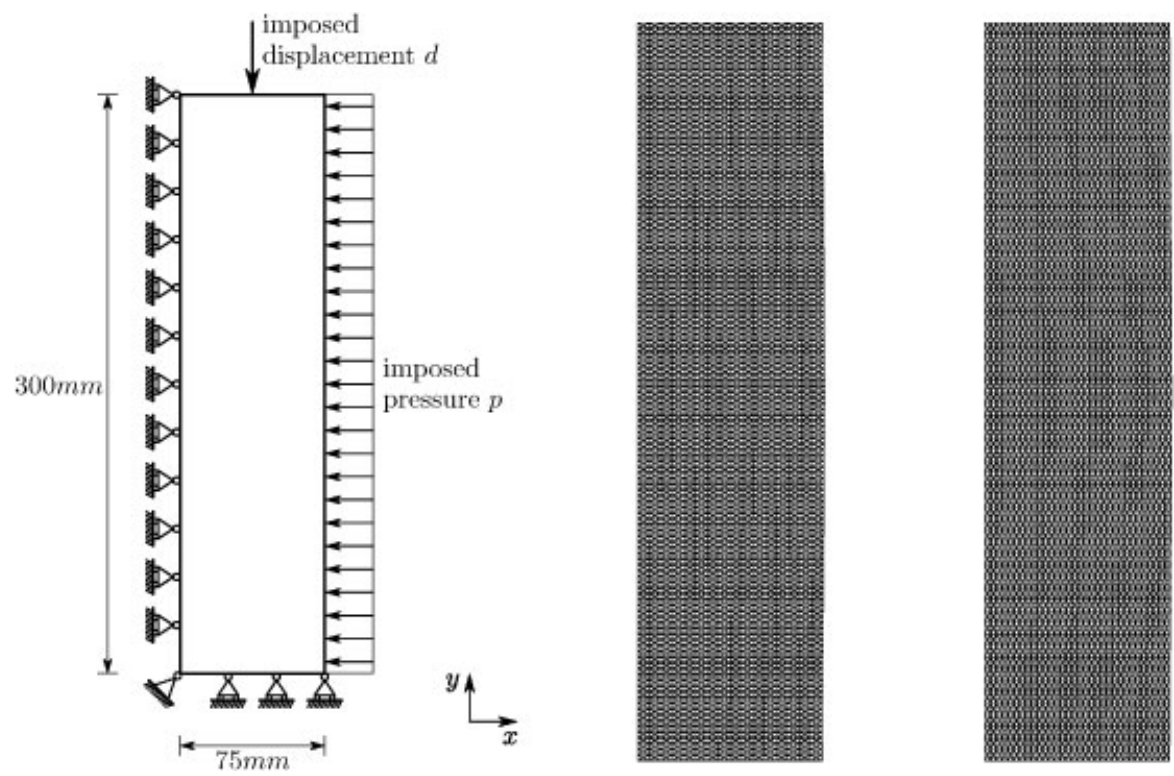

Figure 12. Specimen geometry and FE-meshes used.

which represents about $10^{4}$ times the value of $E_{0}$, when the first plastic deformation occurs. For the Poisson's coefficient of the visco-elastic model the same value as in the elasto-plastic law $(v=0.3)$ is used. 


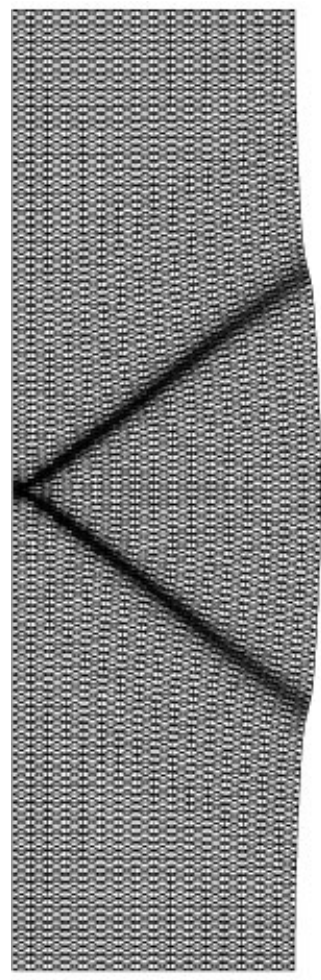

$t=0.95 \mathrm{~s}$

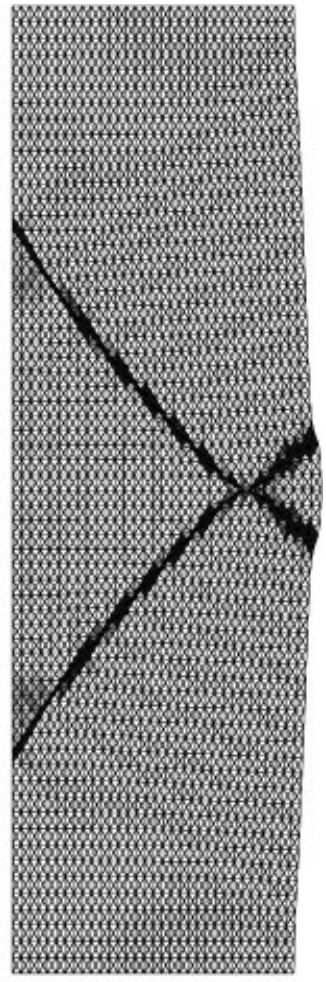

$t=0.90 \mathrm{~s}$

Figure 13. Shear bands obtained with the overlay model and $\beta=17 \times 10^{6} \mathrm{~s}^{-1}$.

In order to test the regularization capabilities of both models in this elasto-plastic model, the process of formation of shear bands in a material specimen is simulated.

As a purely elasto-plastic computation would require too many loading steps, as a consequence of severe time step limitations due to numerical instabilities, the regularization test is performed by comparing a less regularized solution with a more regularized one.

The specimen geometry is presented in Figure 12. In order to localize the deformation, the specimen is not completely prismatic, but has a varying width, which attains its maximum at middle height, with $76 \mathrm{~mm}$ (instead of $75 \mathrm{~mm}$, cf. Figure 12). Pentahedric volume elements with two integration points were used. However, the computation is plain strain, since the displacements in the $z$-direction are blocked. The lateral pressure $p$ is totally applied at first, while the imposed displacement $d$ is applied at the rate of $37.5 \mathrm{~mm} / \mathrm{s}$. This corresponds to the same strain rate as in the one-dimensional example (Figure 11). Loading steps of $3 \times 10^{-3} \mathrm{~s}$ $(0.1125 \mathrm{~mm})$ were used.

A total lagrangian formulation has been used. The material law is considered as a relation between the Green strain and the Piola-Kirchhoff stress. The imposed pressure $p$ corresponds to a Cauchy stress, i.e. it is defined in the deformed configuration of the specimen. 


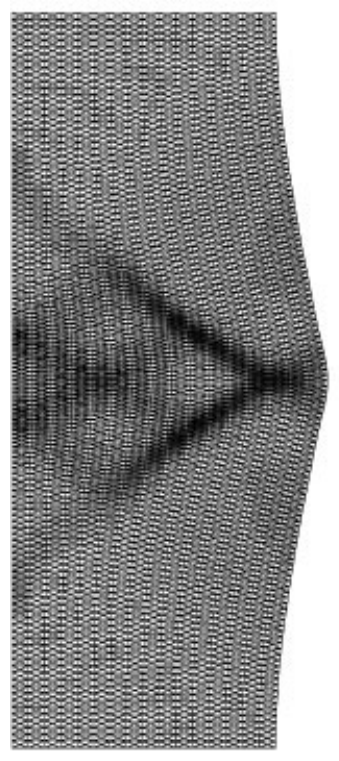

$t=0.95 \mathrm{~s}$

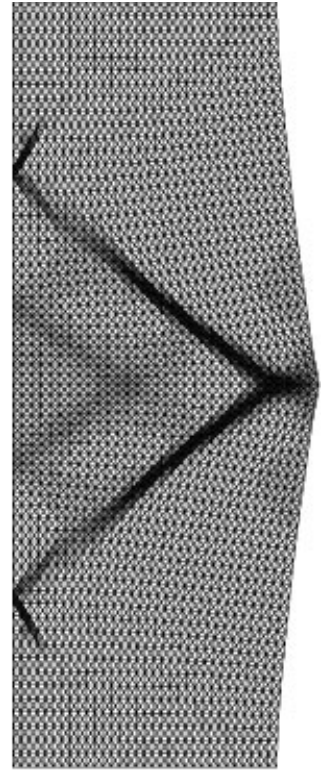

$t=0.90 \mathrm{~s}$

Figure 14. Shear bands obtained with the Duvaut-Lions model and $\beta=200 \mathrm{~s}^{-1}$.

The computation is quasi-static, since no inertial forces are considered. This means that the same results would be obtained with other loading rates, provided that the relaxation modulus varies in such a way, that $\beta \Delta t$ remains constant.

As the less regularized results did not display a relevant dependency on the mesh size (although a fine mesh must be used to allow the shear bands to form), but depend strongly on the mesh orientation, the two meshes presented in Figure 12, with the same size and different orientation have been used.

In Figure 13 the deformation pattern obtained using the overlay model and a value of $\beta=17 \times 10^{6} \mathrm{~s}^{-1}$ in both meshes is presented. It is observed that the shear bands are quite different in the two meshes. It was not possible to go further in the computation, since numerical instability made the results meaningless. The shaded areas represent the elements, in which plastic deformation took place in the considered increment.

In Figure 14 the results obtained by using the Duvaut-Lions model in both meshes, with a relaxations modulus $\beta=200 \mathrm{~s}^{-1}$ are presented. This value was selected by gradually increasing the viscosity (i.e. decreasing $\beta$ ), until a similar deformation pattern in both meshes has been obtained. It was, however, not possible to obtain the same pattern at the same time (cf. Figure 14). A complete regularization could, therefore, not be achieved.

In Figure 15 the results furnished by the overlay model with more viscosity $(\beta=1.7 \times$ $10^{6} \mathrm{~s}^{-1}$ ) are presented. Again, this value of the relaxations modulus was selected by gradually increasing the viscosity.

Also in this case, a complete regularization could not be achieved, since the shear bands do not form at the same time in both meshes, although the difference is smaller than in the 


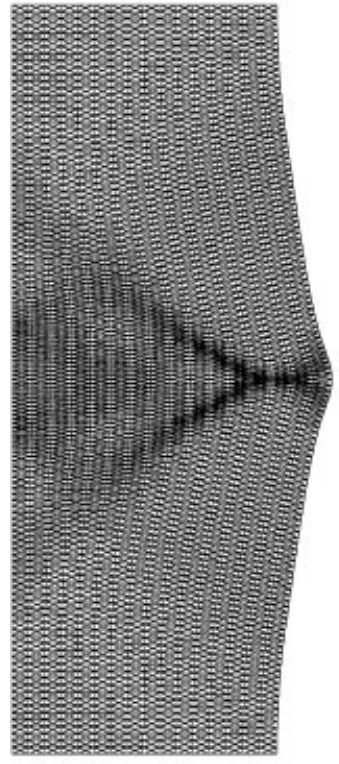

$t=1.94 \mathrm{~s}$

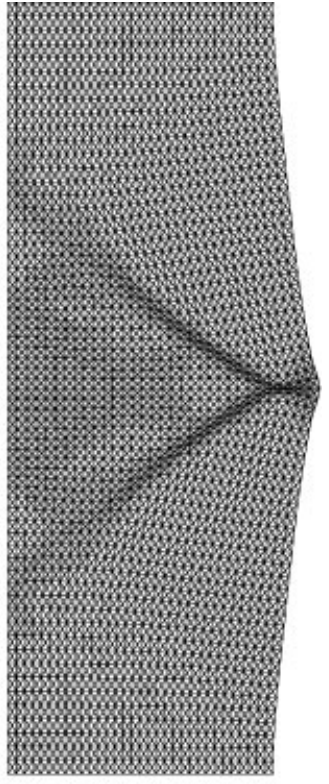

$t=1.76 \mathrm{~s}$

Figure 15. Shear bands obtained with the overlay model and $\beta=1.7 \times 10^{6} \mathrm{~s}^{-1}$.

case of the Duvaut-Lions model. Also, the quality of the regularization is a little better than with the Duvaut-Lions model.

\section{CONCLUDING REMARKS}

The presented examples show that the proposed methodology strongly reduces the dependence of the solution on the mesh alignment, being able to change the direction of the shear bands, so that the same pattern is obtained with both meshes. Furthermore the regularizing capabilities of the proposed model are, at least, as good as the ones displayed by the Duvaut-Lions model, as shown by means of the examples described in Section 3.

The introduction of viscosity allows also a substantial reduction of the computing time, since much larger loading steps may be used. Numerical experiments, without viscosity, have shown that the maximum step size to get a solution in the second example (Cam-Clay) was about 40 times smaller than the used to obtain the solutions presented in Figures 13 and 15.

The presented model has, as compared with the well-known models of Perzyna and DuvautLions, the advantage of being compatible with any integration schema for the inviscid rate equations and to automatically converge to the inviscid solution, when the viscosity goes to zero. Furthermore, its implementation in existing finite element packages for elasto-plasticity requires only the introduction of the constitutive law for the three-dimensional Maxwell model, which can generally be done at the local level. Its capability of regularizing finite element solutions of softening elasto-plasticity has been demonstrated. 
In the FE-computations a lagrangian formulation was used, being assumed that the used constitutive laws represent the relation between Piola-Kirchhoff stresses and Green strains.

\section{REFERENCES}

1. De Borst R, Sluys LJ, Mühlhaus H-B, Pamin J. Fundamental issues in finite element analysis of localization of deformation. Engineering Computations 1993; 10:99-121.

2. Loret B, Prevost JH. Dynamic strain localization in elasto-(visco)-plastic solids, part I. General formulation and one-dimensional examples. Computer Methods in Applied Mechanics and Engineering 1990; 83:247-273.

3. Duvaut G, Lions JL. Les Inequations en Mecanique et en Physique. Dunod: Paris, 1972.

4. Simo JC, Kennedy JG, Govindjee S. Non-smooth multisurface plasticity and viscoplasticity, loading/unloading conditions and numerical algorithms. International Journal for Numerical Methods in Engineering 1988; 26:2161-2185.

5. Wang WM, Sluys LJ, de Borst R. Viscoplasticity for instabilities due to strain softening and strain-rate softening. International Journal for Numerical Methods in Engineering 1997; 40:3839-3864.

6. Simo JC, Hughes TJR. Computational Inelasticity. Springer-Verlag, 2000, ISBN 0-387-97520-9.

7. Argyris J, Doltsinis ISt, Silva VD. Constitutive modeling and computation of non-linear viscoelastic solidspart I: rheological models and numerical integration techniques. Computer Methods in Applied Mechanics and Engineering 1991; 88(2):135-163.

8. Melão Barros MHF, da Silva VD, Serafim JL. Time dependant effects in concrete structures and analysis of the used time-integration algorithm. Second International Conference on Computer Aided Analysis and Design for Reinforced and Prestressed Concrete Structures, vol. 2. Zell am Zee, Austria, 4-6 Abril de 1990; 715-725.

9. Silva VD, Casadei F. An Implementation of the Cam-Clay Elasto-Plastic Model Using a Backward Interpolation and Visco-Plastic Regularization. Technical note No. I.96.239, Institute for Systems, Informatics and Safety, Joint Research Centre, Ispra, Italy, 1996.

10. Wüstenberg H. FEPS 3.3-Finite Element Programming System, Element Library. ICA-Report No. 22, Stuttgart, 1986.

11. Simo JC, Meschke G. New algorithms for multiplicative plasticity that preserve the classical return mappingsapplication to soil mechanics. Third International Conference on Computational Plasticity Fundamentals and Applications, vol. 1, Barcelona, 6-10 April 1992; 765-790. 